\title{
Model Checking for Multiagent Systems: The MABLE Language and its Applications
}

\author{
Michael Wooldridge* Marc-Philippe Huget ${ }^{+}$ \\ Michael Fisher* Simon Parsons ${ }^{\dagger}$ \\ * Department of Computer Science, University of Liverpool \\ Liverpool L69 7ZF, United Kingdom \\ mjw $\mid$ mafecsc.liv.ac.uk \\ + University of Savoie, ESIA-LISTIC \\ B.P. 806, 74016 Annecy CEDEX, France \\ Marc-Philippe.Huget@univ-savoie.fr \\ $\dagger$ Department of Computer \& Information Science \\ Brooklyn College, City University of New York \\ 2900 Bedford Avenue, Brooklyn NY 11210, USA \\ parsonsesci.brooklyn. cuny.edu
}

August 4, 2005

\begin{abstract}
We present MABLE, a fully implemented programming language for multiagent systems, which is intended to support the automatic verification of such systems via model checking. In addition to the conventional constructs of imperative programming languages, MABLE provides a number of agent-oriented development features. First, agents in MABLE are endowed with a BDI-like mental state: they have data structures corresponding to beliefs, desires, and intentions, and these mental states may be arbitrarily nested. Second, agents in MABLE communicate via ACL-like performatives: however, neither the performatives nor their semantics are hardwired into the language. It is possible to define the performatives and the semantics of these performatives independently of the system in which they are used. Using this feature, a developer can explore the design space of ACL performatives and semantics without changing the target system. Finally, MABLE supports automatic verification via model checking. Claims about the behaviour of a MABLE system can be expressed in a linear-time BDI-like logic, and the truth,
\end{abstract}


or otherwise, of these claims can be automatically determined. Following a description of the MABLE language and the language of MABLE claims, we present two case studies to illustrate the language and its use in the verification of multiagent systems. We then describe the key ideas underpinning the current implementation of MABLE. Finally, we survey related work, and discuss some avenues for future research.

\section{Introduction}

We present MABLE, a fully implemented programming language for multiagent systems [45], which is intended to support the automatic verification of such systems via model checking [4]. MABLE is novel in three key respects:

- Agents in MABLE have a mental state consisting of beliefs, desires and intentions; mental states may be nested, so that (for example), one agent is able to have beliefs about another agent's intentions.

- Agents in MABLE communicate using asynchronous message passing, in the style of the FIPA [11] and KQML [22] agent communication languages [7]. However, in MABLE, neither the agent communication language performatives themselves, nor their semantics, are hardwired into the language. Instead, it is possible for a developer to define both the performatives and the semantics of these performatives independently of the system in which they are used. In this way, a developer can explore the design space of ACL performatives and semantics without changing the target system itself.

- MABLE supports automatic verification via model checking [4]. Formal claims about the behaviour of a MABLE system can be expressed in a linear-time BDI-like logic, and the truth or otherwise of these claims can be automatically verified. Thus, in contrast to most logic-based agent programming languages, which perform reasoning at run time, reasoning about the correctness of a MABLE system is carried out at design time (we comment in more detail about the relationship of MABLE to other agent programming languages in section 5).

We emphasise that the MABLE language, as described in this paper, has been fully implemented. The implementation makes use of SPIN [16, 17], a freely available model checking tool for Linear Temporal Logic (LTL). The MABLE compiler takes, as input, a MABLE system together with associated claims about this system (expressed in a BDI-like logic), and generates, as output, both a representation of the MABLE system in PROMELA (the model specification language used by SPIN), and a translation of the BDI logic claims into the LTL logic used by SPIN. SPIN is then invoked, either to automatically verify the truth (or otherwise) of the claims, or else to simulate the execution of the MABLE system, using the PROMELA interpreter provided as part of SPIN. 
The remainder of this paper is structured as follows. We begin by introducing the MABLE language, describe how claims can be made about MABLE programs using a BDI logic called $\mathcal{M O R} \mathcal{A}$, and show how these claims can be automatically verified using MABLE. We then present two detailed case studies, which illustrate the use of MABLE in the verification of multiagent systems. In the first case study, we demonstrate how MABLE can be applied to the problem of verifying that multiagent systems conform to the semantics of a particular agent communication language. While this is a wellknown problem in the multiagent systems literature [41, 42, 26, 34], our work is, to the best of our knowledge, the first to apply model cheching techniques in this area. In the second case study, we present an implementation of the well-known Contract Net task allocation protocol $[36,35]$, and show how properties of this protocol can be verified using MABLE. We proceed to describe the operation of the MABLE compiler, and outline the key techniques used in its implementation. In section 5, we describe the relationship of MABLE to other research on agent programming languages and model checking for multiagent systems. Finally, we present some conclusions, and some pointers to future research.

Throughout the paper, we assume some familiarity with multiagent systems [45], model checking [4], and a basic understanding of conventional programming language design.

\section{The MABLE Programming Language}

MABLE is intended to be used as a language in which programmers can express and verify designs for multiagent systems. As such, one of the aims of MABLE is to provide a collection of constructs which closely resemble those used in conventional programming languages. However, a design requirement of MABLE was that it should be possible to automatically verify properties of systems using model checking: this requirement imposes some significant constraints on the facilities available to programmers in MABLE. For example, at an early stage of MABLE's development, the possibility of providing a JAVA-like object-oriented programming model was investigated. However, to provide such features would have necessitated the implementation of an objectoriented interpreter (similar to the JAVA virtual machine) in the modelling language of the target model checker, resulting in a dramatic blow-up in the size and complexity of models. The resulting state space explosion would almost certainly make the verification of systems impossible. For this reason, it was decided instead to provide a C-like imperative language, enriched by a number of key agent-oriented constructs. In particular, the key agent oriented features provided by MABLE over and above the basic system modelling facilities available in model checking systems such as SPIN $[16,17]$, SMV [24], and MOCHA [1] are as follows:

- Agents in MABLE have a mental state consisting of beliefs, desires and intentions. 
- Agents in MABLE communicate using ACL performatives, and it is possible for a developer to define both the performatives and the semantics of these performatives independently of the system in which they are used.

In addition, MABLE provides "syntactic sugar" for many programming language features that are not provided as standard in most model checker system modelling languages (which tend to be rather low-level guarded command languages). In particular, MABLE provides the full range of iteration, sequence, and selection operations familiar from languages such as C and JAVA, C-like structure type declarations, and several high level synchronisation constructs. Note that we comment on the relationship of MABLE to other agent programming languages in section 5.

Over the past two decades, many logics and related formalisms have been proposed for representing and reasoning about multiagent systems, of which Rao and Georgeff's BDI logics are perhaps the best known [29, 44]. Ideally, then, we would like to take a BDI logic such as $\mathcal{L O} \mathcal{R} \mathcal{A}$ (described in [44]) off the shelf, and develop verification tools that would allow us to determine whether or not systems implement specifications expressed in this logic. However, it is well-known that the link between such logics and implemented systems is informal at best. There is, in general, no systematic way of associating models for such logics with implemented systems: this is known as the problem of computational grounding [40, 43]. So, what we have done instead is to develop a slightly simplified and cut-down version of $\mathcal{L O R} \mathcal{A}$, known as $\mathcal{M O R} \mathcal{A}$, in which claims about systems can be expressed. We have then developed a mapping from this BDI-like logic to the Linear Temporal Logic used by the SPIN model checker; in this way, we can leverage existing model checking tools - and in particular, SPIN $[16,17]$ — to verify properties of MABLE systems.

In summary, then, a MABLE system consists of:

- a number of agent definitions and associated type and variable declarations, where each agent is programmed using the MABLE agent programming language;

- explicit semantics for the performatives used in the system;

- a number of formal claims about the system.

In the subsections that follow, we briefly describe these three elements. We begin with a survey of the agent programming language; we then describe the way in which the semantics of communication language performatives may be defined, and the use of claims in MABLE systems.

\subsection{The Agent Programming Language}

For a programmer, the core component of MABLE is of course the agent programming language. As noted above, this language is in essence a C-like imperative language, enriched by agent-oriented features. The concrete syntax of the conventional program 
constructs in MABLE is based on that of C/JAVA, and so we will not give a detailed description here. Instead, we will give an overview of the main language features, focussing on those that are unique to MABLE.

\section{Agent declarations and initialisation}

Agents are declared via the agent keyword, followed by the agent's name (which must be unique), and the body of the agent. At startup, agents are invoked in the order in which they are declared; an agent terminates when it reaches the end of its code body. (Agents are not functions, and therefore do not return values; however, there is a function facility in MABLE, described below.) There is at present no way of invoking multiple copies of the same agent in MABLE, or of passing initialisation parameters to agents. However, it is possible for programmers to declare an explicit init section, which can be used for initialisation of system parameters. The init section is executed before any agent is invoked.

\section{Beliefs, desires, and intentions}

Perhaps the most obvious way in which MABLE differs from conventional programming languages is that the processes - agents - in a MABLE system have explicitly represented data structures corresponding to beliefs, desires, and intentions [29, 44]. These mental states can be nested, so that (for example) an agent can have beliefs about another agents intentions. Intuitively, an agent's beliefs are the information it has about the environment; these beliefs may be incorrect. An agent's desires and intentions come into play primarily when the agent is involved in communication.

In MABLE an agent's mental states are attitudes to the variables in the system. So, for example, agent one might have the belief that agent two intends that variable $x$ has value greater than 10. Programmers can directly refer to an agent's mental state by means of modal expressions, or modalities. The intended meaning of the modality ( $m$ $a g c$ ) is that agent $a g$ has attitude $m$ (where $m$ is believe, desire, or intend) towards the condition (predicate) $c$. The identifier $a g$ must be the name of an agent in the system, and $c$ must be a MABLE condition. The following is thus a legal modal expression in MABLE.

$$
\text { (believe agent1 }(a==10) \text { ) }
$$

Suppose this expression is evaluated by agent 2 . Then it will "true" if agent 2 believes that agent 1 believes that $a==10$. Mental states are implemented in MABLE as nested sets of facts (in the style of [18]): to evaluate this expression, agent 2 will check in its belief set, and inside this set will look for the set of facts representing agent 1 's beliefs. If it finds that $a=10$ in this set (i.e., the set representing agent2's beliefs about agent 1's beliefs), then the expression will evaluate to "true".

As modalities are themselves conditions, they may be arbitrarily nested. For example, the following is also a legal modal expression in MABLE: 


\section{(intend agent1 (believe agent2 ( $a==10)$ ))}

Again, suppose this expression is evaluated by agent 1: it will evaluate to "true" if agent 2 believes that agent 1 intends that agent 2 believes that $a==10$.

In order to directly manipulate beliefs, desires, and intentions, MABLE provides assert and retract statements. These statements take a single argument - a condition - and behave rather like the PROLOG assert and retract predicates [5]. Thus, for example, consider the following MABLE statement.

assert((intend agent2 $\mathrm{x}==10)$ )

The effect of this statement is to make the agent executing it subsequently believes that agent 2 intends that variable $\mathrm{x}$ has the value 10 .

An agent's mental state can also be modified in two other ways. First, an agent's beliefs can be changed by assignment and observe statements, as described in the following section. Second, communication actions may change an agent's mental state, as defined by in the performative semantics; we shall see how this works in section 2.2.

\section{Types, variables, expressions, and assignments}

MABLE supports C-style structure and array declarations, which may be composed in terms of integer and boolean data types. Variables in MABLE may be local, shared, or global. A local variable is private to an individual agent. A shared variable is declared outside an agent, and is visible to all agents in the system: all agents implicitly have access to shared variables, and moreover all agents can write to shared variables.

Like shared variables, global variables are also declared outside the scope of an agent. However, there is an important difference between global and shared variables. All agents implicitly know the value of shared variables; all agents have complete, correct, up-to-date beliefs about the value of shared variables. With global variables, however, the situation is slightly different. While all agents may still access global variables, they must explicitly request access in order to discover their value. They do this by executing a MABLE observe statement. The observe construct can thus be viewed as a sensing action. When an agent executes an observe instruction, its beliefs about the value of the variable it observes are synchronised with the true value of this variable. However, if the value of the variable is subsequently changed, then the agent will not necessarily be aware of this - its beliefs about the value of the variable may thus become "out of date". If an agent modifies the value of a global variable, then its beliefs about the value of this variable are similarly synchronised. Once again, however, its beliefs may become out of date if the value of this variable is changed by some other agent.

The syntax of variable declarations is broadly the same as C/JAVA. Expressions and assignment statements in MABLE also follow the conventions of C/JAVA; all the arithmetic operators that one would expect to find in an imperative language are present. 


\section{Conditional expressions}

Conditional (boolean) expressions in MABLE may be constructed from expressions via the usual relational operators $(<,>,==, \ldots)$. However, MABLE also permits conditions to contain modalities, as described above: in particular, belief, desire, and intention modalities.

\section{Selection}

MABLE contains the selection statements that one would expect from an imperative programming language - if... else and multi-way selection via switch statements. However, as noted earlier, the conditions in these constructs may contain belief, desire, and intention modalities. For example, suppose that agent agent 1 was executing the following statement.

$$
\text { if (intend agent2 }(a==10) \text { ) XYZ; }
$$

Then, in this case, agent 1 would execute statement XYZ if it believed that agent 2 intended that $\mathrm{a}==10$.

\section{Loops}

MABLE provides all the loop constructs found in C/JAVA (i.e., for, while, and do), and the syntax follows the conventions of these languages. There is an additional looplike construct, which is not found in languages like C/JAVA: await. This construct implements an idle (non-busy) wait construct: it takes a single parameter, a condition, and the effect is that the agent executing the await is suspended until it believes the condition is satisfied.

\section{Communication}

MABLE provides two built-in communication primitives: send and receive. Their syntax is as follows:

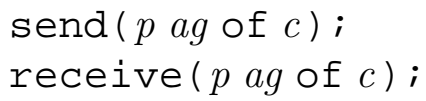

where $p$ is the performative, $a g$ is the name of an agent (the recipient of the message, in the case of send, the sender in the case of receive), and $c$ is the message content, which must be a MABLE conditional expression. For example, the following is a syntactically acceptable send statement.

$$
\text { send (inform agent } 2 \text { of }(x==10)) \text {; }
$$

(Note that of is just syntactic sugar, which play no other role.) The following is also a legal send statement. 
send (informagent 2 of (intend agent $3 \times==10)$ );

The effect of communication is to change the mental state of the recipient of the message. However, the actual effect that a message has is not defined within the program. It is defined externally, in the semantic definition file, as described below.

Note that message delivery is guaranteed, but is asynchronous: receive statements block until a message is available to be received, although send statements do not block. Broadcast message passing is not currently supported.

\section{Synchronisation}

In order to allow agents to synchronise their activities, MABLE provides facilities for enforcing mutual exclusion over critical sections of code. A MABLE system can contain an arbitrary number of locks, each of which is identified by a unique name. Sections of code can be wrapped in a lock statement, associated with a particular named lock. Only one agent can access a lock at any given time. When an agent comes across a locked section of code, it suspends until the associated lock is free, at which point it obtains the lock in an atomic operation, and enters the critical section; when it exits the code, the lock is released.

In addition, MABLE enables an agent to obtain exclusive access to a shared variable via the read construct. As long as an agent uses the read construct, it is impossible for other agents to access the variable locked with this construct. The lock is released when the agent exits the read block.

\section{Functions}

MABLE provides functions as a structuring mechanism for programs, and the syntax used for defining and invoking functions is again based on that of C. Functions may take arbitrary parameters, although at present may only return integer (int) values. All functions have global scope, and can be invoked either by agents or by other functions.

\section{I/O}

MABLE is intended primarily as a framework for model checking and, as such, there are critical limitations on the I/O facilities available in the language. Contemporary model checking techniques are focussed around finite state and hence closed systems. Thus it is not possible for a MABLE system to obtain input at run time from the outside environment. Where this is desired, a solution is to model the environment as an agent that provides appropriate input to other agents. However, a print statement is provided as a means to display output from MABLE. 


\section{Pre-processing}

Before processing source code, the MABLE compiler runs the standard C pre-processor over files. This makes it possible to use all the pre-processor directives available in C:

- macro definitions, via the \#define directive;

- textual file inclusion, via the \# include directive;

- conditional compilation, via the \# if ...\#endif directive.

\subsection{Communication in MABLE}

A key component of the current version of MABLE is that programmers can define their own semantics for performatives, separately from a program in which these performatives are used. The formalism we use for defining semantics is a STRIPS-style pre/post-condition model, in the way pioneered for the semantics of speech acts by Cohen and Perrault [6], and subsequently applied to the semantics of the KQML [19] and FIPA languages [11]. Thus, to give a semantics to performatives in MABLE, a user must define, for every such communicative act, a pre-condition and a post-condition. Formally, the semantics for a communicative act $C A$ is defined as a pair $\left\langle C A_{\text {pre }}, C A_{\text {post }}\right\rangle$, where $C A_{\text {pre }}$ is a condition (a MABLE predicate), and $C A_{\text {post }}$ is a condition to be asserted. The basic idea is that, when an agent executes a send statement with performative $C A$, this message will not be sent until $C A_{\text {pre }}$ is true. When an agent executes a receive statement with performative $C A$, then when the message is received, the assertion $C A_{\text {post }}$ will be made true.

By default, the MABLE compiler looks for performative semantics in a file that is named mable.sem. A mable. sem file contains a number of performative definitions, where each performative definition has the following structure:

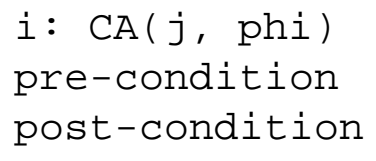

where $i, j$ and phi are bound to the sender, recipient, and content of the message respectively, and CA is the name of the performative. The following two lines define the pre-condition and post-condition associated with the communicative act CA.

The way in which pre-conditions are used is as follows. Suppose an agent agent 1 executes the following statement

send ( $P$ agent2 of $C$ )

where the semantics of the performative $\mathrm{P}$ are defined as follows. 


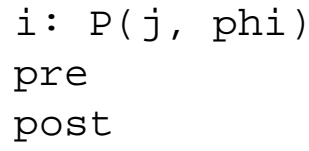

Then the agent agent 1 will suspend (i.e., enter a non-busy wait state) until the condition pre is believed to be true by agent 1 , at which point it will send the message. Notice that it is possible to define the pre-condition of a performative simply as " 1 ", i.e., a logical constant for truth. In this case, the agent executing the send will never be suspended - the message will be sent immediately.

With respect to the post-condition, the idea is that once a message is received, the corresponding post-condition will be made true. Notice that post-conditions in a mable. sem file do not correspond to the "rational effect" parts of messages in FIPA semantics [9]; we elaborate on the distinction below.

Here is a concrete example of a mable. sem performative semantic definition:

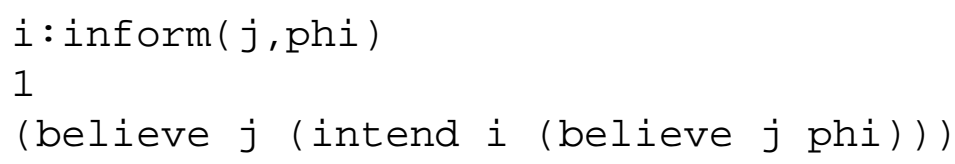

This says that the sender of a message will always send an in form message directly; it will not wait to check whether any condition is true. It also says that when an agent receives an inform message, it will subsequently believe that the sender intends that the receiver believes the content.

By disconnecting the semantics of a communicative act from a program that carries out such an act, we can experiment to see the effect that different kinds of semantics can have on the same agent.

\subsection{Claims}

Another key component of MABLE is that agents may be augmented with formal claims about their behaviour. Claims are expressed in $\mathcal{M O R} \mathcal{A}$, a subset of the $\mathcal{L O} \mathcal{R} \mathcal{A}$ BDI logic introduced in [44]. These claims can be automatically checked, by making use of the underlying SPIN model checker. If the claim is disproved, then a counter example is provided, illustrating why the claim is false.

A claim is introduced outside the scope of an agent, with the keyword claim followed by a $\mathcal{M O R} \mathcal{A}$ formula, and terminated by a semi-colon. The formal syntax of $\mathcal{M O R} \mathcal{A}$ claims is given in Figure 1. The language of claims is thus that of quantified linear temporal BDI logic, with the dynamic logic style "happens" operator, similar in intent and role to that in $\mathcal{L O R} \mathcal{A}$ [44]. The operators of $\mathcal{M O} \mathcal{R} \mathcal{A}$ have the following intuitive meaning. First, any valid MABLE condition is an acceptable $\mathcal{M O R} \mathcal{A}$ formula, and thus it is possible to express conditions over all shared and global variables of a system. MABLE also supports the LTL operators of SPIN, as follows. First, [ ] ("always") is the always in the future operator: thus a formula [ ] P asserts that P will be true now 


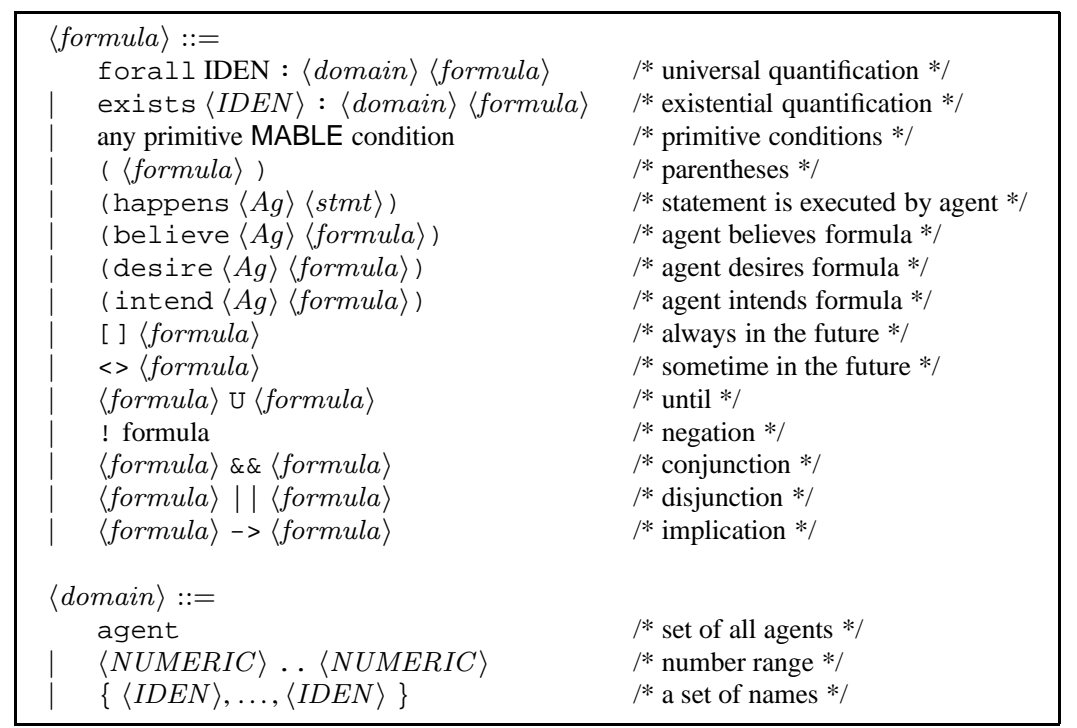

Figure 1: The syntax of $\mathcal{M O R} \mathcal{A}$ claims.

(i.e., in the present state) and forever (i.e., in all future states). The $<>P$ ("sometimes P") construct means "eventually, P will be true". In other words, P will either be true in the present state, or at some future state. (The <> construct does not assert the unique existence of such a state: it may be that $\mathrm{P}$ is several times in the future, or even that $P$ is always true.) The $U$ ("until") operator is a binary operator, and a formula $P \quad U Q$ asserts that $\mathrm{P}$ is true now, and will remain true until $\mathrm{Q}$ is true.

$\mathcal{M O} \mathcal{R} \mathcal{A}$ supports quantification over finite domains, and in particular, over the following sets:

- agents (e.g., "every agent believes $\varphi$ ”);

- finite sets of objects (e.g., enumeration types); and

- integer number ranges.

The believe, desire, and intend operators make it possible to make claims about agents' mental states. These constructs have the same interpretation in $\mathcal{M O R} \mathcal{A}$ claims as in conditionals, as described above.

To better understand how these constructs may be combined to make claims, consider the following informal examples.

First, suppose we want to express the fact that, whenever agent $a_{1}$ believes the reactor failed, then $a_{1}$ intends that $a_{2}$ believes the reactor failed (i.e., $a_{1}$ wants to communicate this to $a_{2}$ ).

We can express such a property directly as the following $\mathcal{M O R} \mathcal{A}$ claim.

claim 


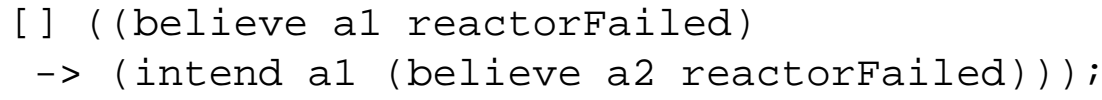

The outer [ ] is the temporal "always" operator, and ensures that this property is checked in every possible state that the system enters. Here, the variable reactorFailed is assumed to be boolean.

Next, suppose we want to say that if some agent wants agent $a_{2}$ to believe that the reactor has failed, then eventually, $a_{2}$ will believe it has failed.

This translates directly into the following $\mathcal{M O R} \mathcal{A}$ claim.

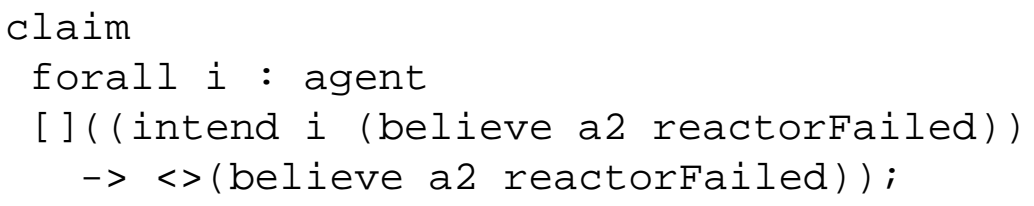

Next, we describe the "happens" construct. Recall that the syntax of this construct is as follows:

\section{(happens ag stmt)}

where $a g$ is the name of an agent and stmt is a MABLE program statement. This predicate will be true in a state whenever the next statement that agent $a g$ will perform is stmt. Consider the following concrete example.

claim

[] ( (happens a1 $\mathrm{x}=10$;)

$-><>($ believe al $\mathrm{x}==10))$;

This claim says that, whenever the next statement to be executed by agent a 1 is the assignment $\mathrm{x}=10 ;$, then eventually, a 1 believes that variable $\mathrm{x}$ has the value 10 . Notice that the semi-colon is part of the assignment program statement, and must therefore be included in the happens construct. Also recall that a single equals sign in MABLE is an assignment, while a double equals sign is the equality predicate. As we will see below, the happens construct plays a key role in our approach to ACL compliance verification.

Finally, let us consider exactly how claims are checked by the MABLE compiler. Suppose that a system contains a single claim, $\varphi$, and that the programmer invokes the MABLE compiler signalling that this claim should be checked ${ }^{1}$. Then MABLE will systematically generate, (by means of the SPIN system), every possible computation $c=s_{0}, s_{1}, s_{2}, \ldots$ of the system. Each computation $c$ corresponds to a model for $\mathcal{M O R} \mathcal{A}$, and the claim $\varphi$ will either be true or false when interpreted in this model. So, for every computation $c$, MABLE will check whether this computation satisfies $\varphi$; if

\footnotetext{
${ }^{1}$ The default behaviour of the MABLE compiler is to ignore claims; a user indicates to the compiler that claims should be checked by means of a command line argument.
} 
MABLE ever encounters a computation $c$ such that $c \not \models \varphi$, then MABLE halts, and reports $c$ as a counterexample to the claim $\varphi$. If no such computation is found, then MABLE (or more accurately, SPIN!) will continue until it has exhaustively examined the entire space of possible computations.

\section{Two Case Studies}

This section presents two detailed case studies. The first case study demonstrates how MABLE can be used to verify that agents correctly implement the semantics of an agent communication language [41]. In the second case study, we show how MABLE can be used to implement the Contract Net protocol $[36,36]$, and we show how properties of this protocol can be established via model checking.

\subsection{Verifying Compliance with respect to ACL Semantics}

In this section, we will show how conformance to the pre-condition and rational effect parts of ACL semantics can be verified with MABLE. We also show how, by varying the semantics of performatives, we achieve different results for the same agent programs. We begin with a brief introduction to the ACL verification problem.

The need for agents to be able to inter-operate has led to the development of several standardised agent communication languages (ACLs) [22, 10]. However, in order to gain acceptance, particularly for sensitive applications such as electronic commerce, it must be possible to determine whether or not any system that claims to conform to an ACL standard actually does so. We say that an ACL standard is verifiable if it enjoys this property. FIPA - currently the main standardisation body for agent communication languages - recognises that "demonstrating in an unambiguous way that a given agent implementation is correct with respect to [the semantics] is not a problem which has been solved" [10], and identify it as an area of future work. (Checking that an implementation respects the syntax of an ACL such as that proposed by FIPA is, of course, trivial.) If an agent communication language such as FIPA's is ever to be widely used - particularly for such sensitive applications as electronic commerce - then such compliance testing (verification) is important. However, the problem of compliance testing is not actually given a concrete definition by FIPA, and no indication is given of how it might be done.

In [41], the verification problem for agent communication languages was formally defined for the first time. It was shown that verifying compliance to some agent communication language reduced to a verification problem in exactly the sense that the term in used in theoretical computer science. To see what is meant by this, consider the semantics of FIPA's inform performative [10, p25]: 


$$
\begin{aligned}
& \langle i, \operatorname{inform}(j, \varphi)\rangle \\
& \mathrm{FP}: \quad B_{i} \varphi \wedge \neg B_{i}\left(B_{i} \varphi \vee U_{j} \varphi\right) \\
& \mathrm{RE}: \quad B_{j} \varphi
\end{aligned}
$$

Here $\langle i, \operatorname{inform}(j, \varphi)\rangle$ is a FIPA message: the message type (performative) is inform, the content of the message is $\varphi$, and the message is being sent from $i$ to $j$. The intuition is that agent $i$ is attempting to convince (inform) agent $j$ of the truth of $\varphi$. The FP and RE components define the semantics of the message: FP is the feasibility pre-condition, which states the conditions that must hold in order for the sender of the message to be considered as sincere; RE is the rational effect of the message, which defines what a sender of the message is attempting to achieve. The $B_{i}$ is a modal logic connective for referring to the beliefs of agents (see e.g., [14]); Bif is a modal logic connective that allows us to express whether an agent has a definite opinion one way or the other about the truth or falsity of its parameter; and $U$ is a modal connective that allows us to represent the fact that an agent is "uncertain" about its parameter. Thus, an agent $i$ sending an inform message with content $\varphi$ to agent $j$ will be respecting the semantics of the FIPA ACL if it believes $\varphi$, and it it not the case that it believes of $j$ either that $j$ believes whether $\varphi$ is true or false, or that $j$ is uncertain of the truth or falsity of $\varphi$.

It was noted in [41] that the FP acts in effect as a specification or contract that the sender of the message must satisfy if it is to be considered as respecting the semantics of the message: an agent respects the semantics of the ACL if, when it sends the message, it satisfies the specification. Although this idea has been understood in principle for some time, no serious attempts have been made until now to adopt this idea for ACL compliance testing.

Note that a number of other approaches to ACL compliance testing have been proposed in the literature. Although it is not the purpose of this paper to contribute to this debate, we mention some of the key alternatives. Pitt and Mamdani defined a protocol-based semantics for ACLs [26]: the idea here is that the semantics of an ACL are defined in terms of the way that they may be used in the context of larger structures, i.e., protocols. Singh championed the idea of social semantics: the idea here being that an ACL semantics should be understood in terms of the observable, verifiable changes in social state (the relationships between agents) that use of a performative causes [34].

We begin with a running example that we will use in the following sections to illustrate the approach. The example employs the "inform" performative, which is one of the two key performatives in the FIPA framework [11]. (The other is "request", which can be dealt with using the same techniques.) The MABLE code for this example is given in Figures 2 and 3. Two agents have several beliefs and they simply send messages among themselves communicating these beliefs. The selection of the message to be sent is carried out non-deterministically, via the choose construct. The insertion of these beliefs in agents' mental state is done through the as sert statements. 


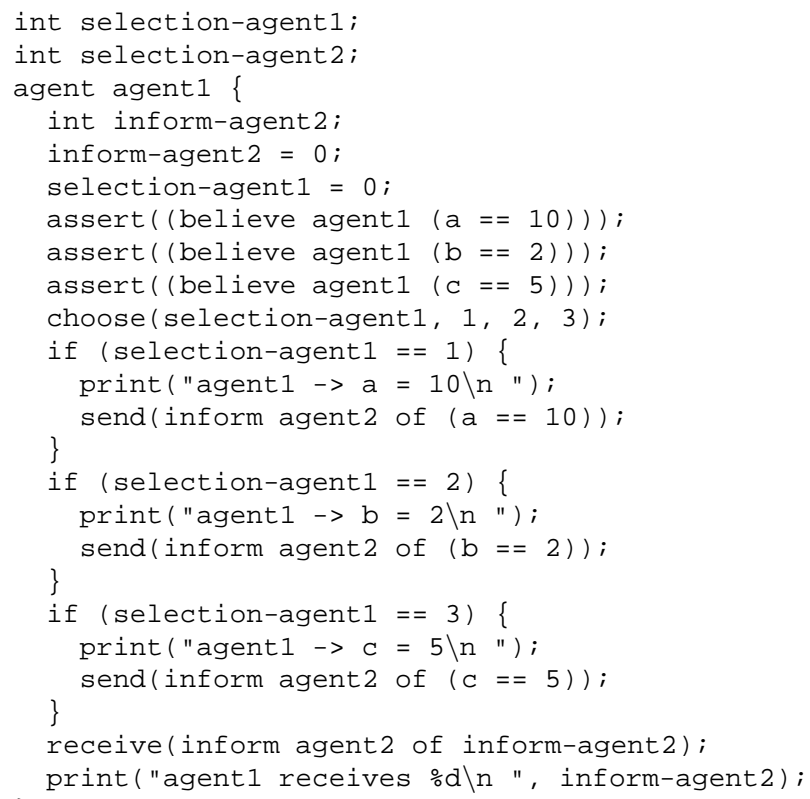

Figure 2: The base example (agent 1).

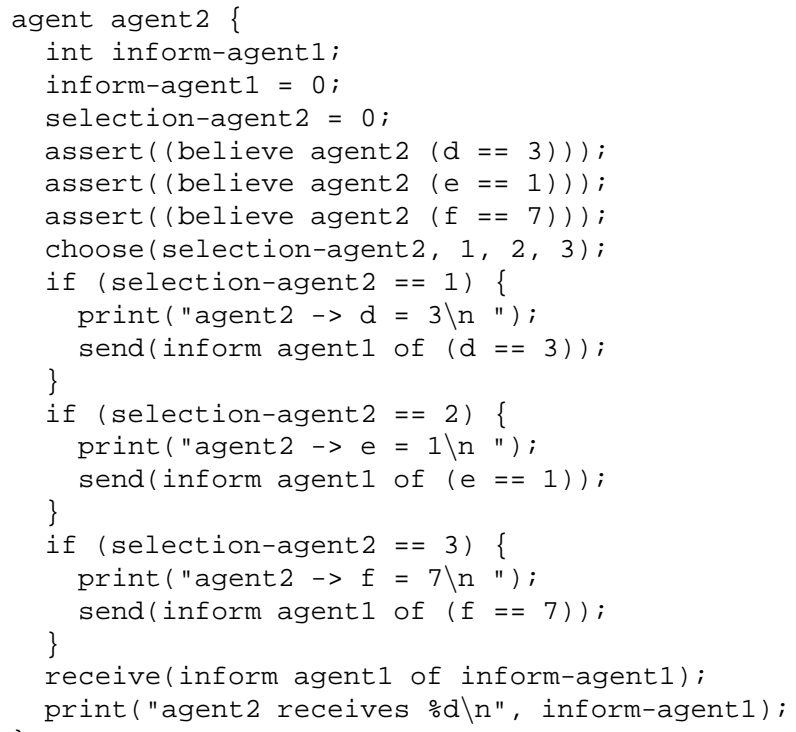

Figure 3: The base example (agent 2).

\section{Verifying Performative Pre-Conditions}

Verifying pre-conditions means verifying that agents satisfy the pre-condition part of an ACL performative's semantics whenever they send the corresponding message. 
There are essentially two possibilities with respect to pre-conditions: either agents are sincere (they only ever send an inform message if they believe its content), or else they are not (in which case they can send a message without checking to see whether they believe it). We can use MABLE'S ACL semantics to define these two types of agents. Consider first the following mable. sem definition.

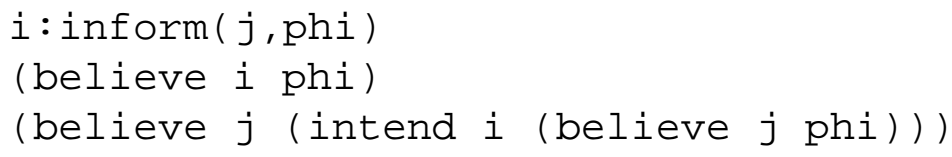

This says that the pre-condition for an inform performative is that the agent believes the content phi of the message. By defining the semantics in this way, an agent will only send the message if it believes it. (If the sender never believes the content, then its execution is indefinitely postponed.)

By way of contrast, consider the following mable. sem definition of the inform performative.

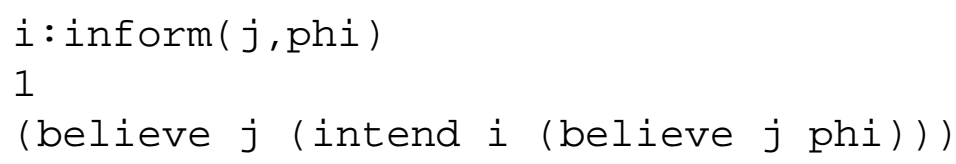

Here, the guard to the send statement is 1 , which, as in languages such as C, is interpreted as a logical constant for truth. Hence, the pre-condition test will always succeed, and the message send statement will always be enabled, irrespective of whether or not the agent actually believes the message content. Notice that this second case is actually the more general one, which we would expect to find in most applications.

The next stage is to consider the process of actually checking whether or not agents respect the semantics of the language; of course, if we enforce compliance by way of the mable.sem file, then we would hope that our agents will always satisfy the semantics. But it is also possible that an agent will respect the semantics even though they are not enforced by the definition in mable. sem. (Again, this is in fact the most general case.)

For FIPA-style inform performatives, the property we want is that, whenever agent $i$ sends an inform message to agent $j$ with content $\varphi$, then $i$ believes $\varphi$. Now, given the enriched form of MABLE claims that we described above, we can directly encode this formula in $\mathcal{M O R} \mathcal{A}$, as follows:

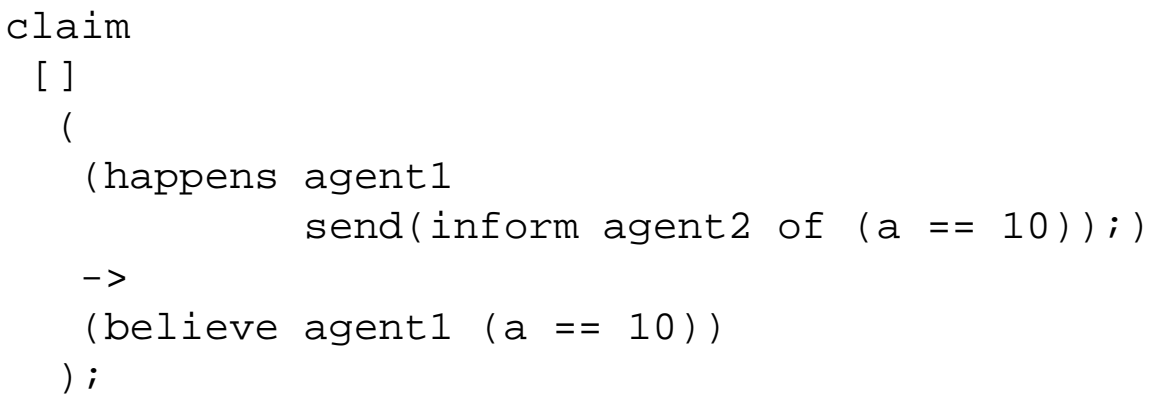


This claim will hold of a system if, whenever the program statement

send(inform agent2 of $(a==10))$;

is executed by agent 1 , then in the system state from which the send statement is executed, agent 1 believes that $a==10$.

We can insert this claim into the system given in Figures 2 and 3, and use MABLE to check whether it is valid. If we do this, then we find that the claim is indeed valid; inspection of the code suggests that this is what we expect.

Verifying pre-conditions also implies that we ensure agents do not inform other agents about facts that they do not believe. In our running example, we simply have to remove the line

assert ((believe agent1 $(\mathrm{a}==10)))$;

and then set the pre-condition of the inform to 1 (i.e., true) in the mable.sem file, and check the previous claim. The claim is now not valid, as agent 1 informs agent 2 about something it does not believe.

\section{Verifying Performative Rational Effects}

We consider an agent to be respecting the semantics of an ACL if it satisfies the specification defined by the pre-condition part of a message whenever it sends the message [41]. The rational effect part of a performative's semantics defines what the sender of the message wants to achieve by sending it; but this does not imply that sending the message is sufficient to ensure that the rational effect is achieved. This is because the agents that receive messages are assumed to be autonomous, exhibiting control over their own mental state. Nevertheless, it is useful to be able to determine, in principle, whether an agent respects the rational effect part of an ACL semantics or not, and this is the issue we discuss in this section.

We will consider two cases: credulous agents and sceptical agents. Credulous agents correspond to agents that always believe the information sent by other agents. We can directly define credulous agents via the following mable. sem file.

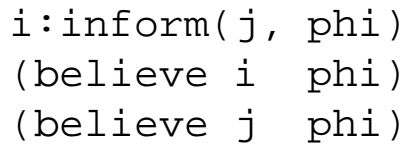

This says that the recipient $j$ of an inform message will always come to believe the contents of an inform message.

Sceptical agents are those that believe that the sender intends that they believe the information, but do not necessarily come to directly believe the contents of the message. 


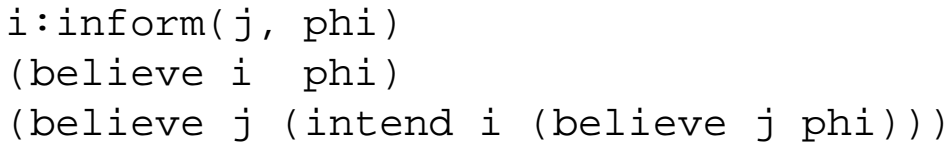

We can directly define a $\mathcal{M O R} \mathcal{A}$ claim to determine whether or not an agent that is sent a message eventually comes to believe it.

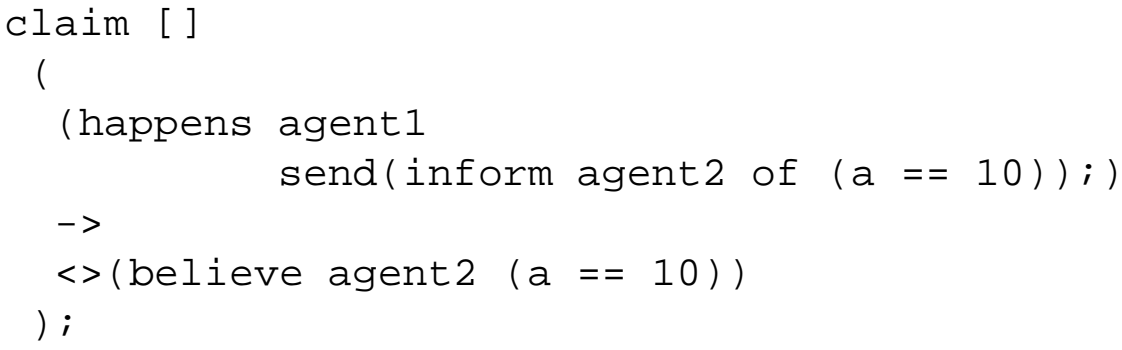

This claim is clearly valid for credulous agents, as defined in the mable.sem file given above; running MABLE with the example system immediately confirms this.

Of course, the claim may also be true for sceptical agents, depending on how their program is defined. We can directly check whether or not a particular sceptical agent comes to believe the message it has been sent, with the following claim:

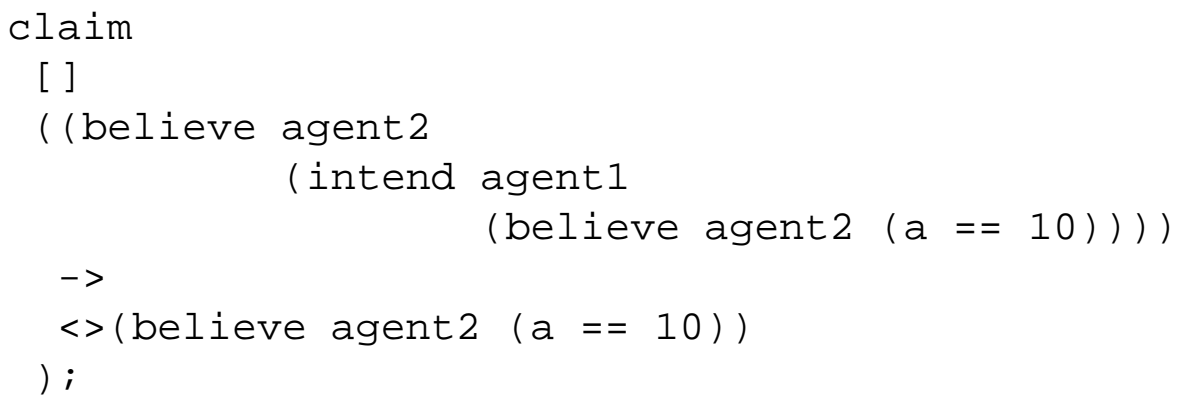

\subsection{The Contract Net Protocol}

In the section, we will show how the well-known Contract Net protocol can be implemented using MABLE $[36,35]$, and then demonstrate how properties of this implementation may be verified using MABLE claims.

The Contract Net Protocol was proposed by Smith [36, 35] as a mechanism for task allocation in distributed problem solving systems. The idea of this protocol is that one agent (the initiator of the interaction) has a task to carry out, but requires cooperation for this task - either because the task requires resources that are unavailable to the initiator, or else because a cooperative solution will be preferred to a non-cooperative one. The initiator takes the role of task manager and broadcasts an announcement of the task to other agents. In general, the task announcement specifies the properties of the task - quality of service parameters, and any other information that a potential 
bidder may require to determine whether or not to submit a bid to carry out the task. In our implementation, the task announcement defines the skills required to solve the task (its "weight”).

Agents receiving a task announcement have several choices. They can either submit a bid for the task (e.g., specifying a price for carrying the task out), or else they can choose not to bid. When the task manager has the answers of the bidders, it can choose a bidder, to whom it awards the task.

The implementation of the Contract Net protocol in MABLE represents about 250 lines of code. It contains three agents: the task manager and two bidding agents. Additionally two functions are declared.

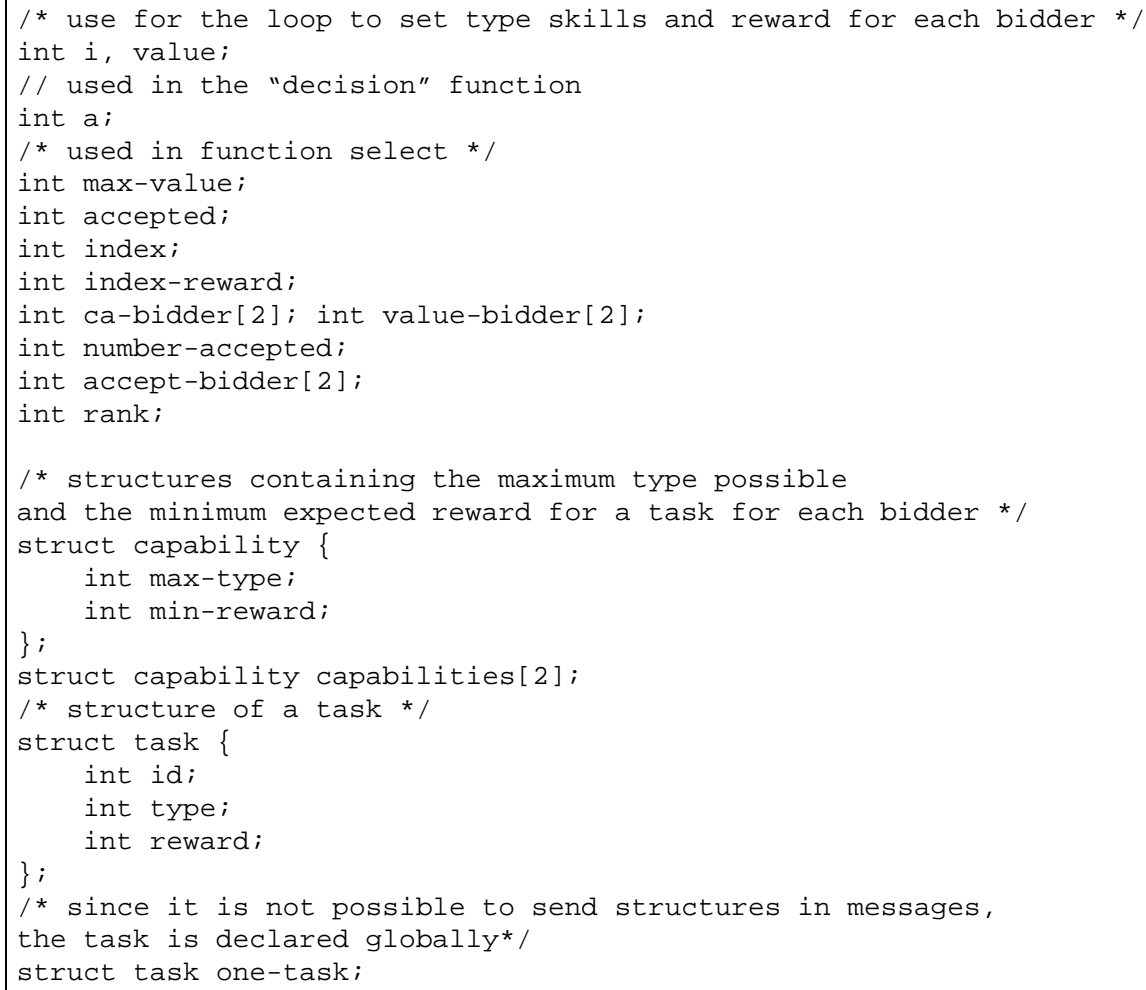

Figure 4: Contract Net Protocol Declarations.

The variable declarations for the system are shown in Figure 4. The init section for the system is shown in Figure 5. In this section, we first define the task, then we define bidders' parameters: the maximum size of task they can perform, and their expected reward for a task. These values are set non-deterministically, through the choose construct.

The implementation of the task manager is given in Figure 6. The task manager first informs the two bidders that a task has to be performed, and then waits for an answer. In our model, bidders are obliged to answer either with an acceptance or a 


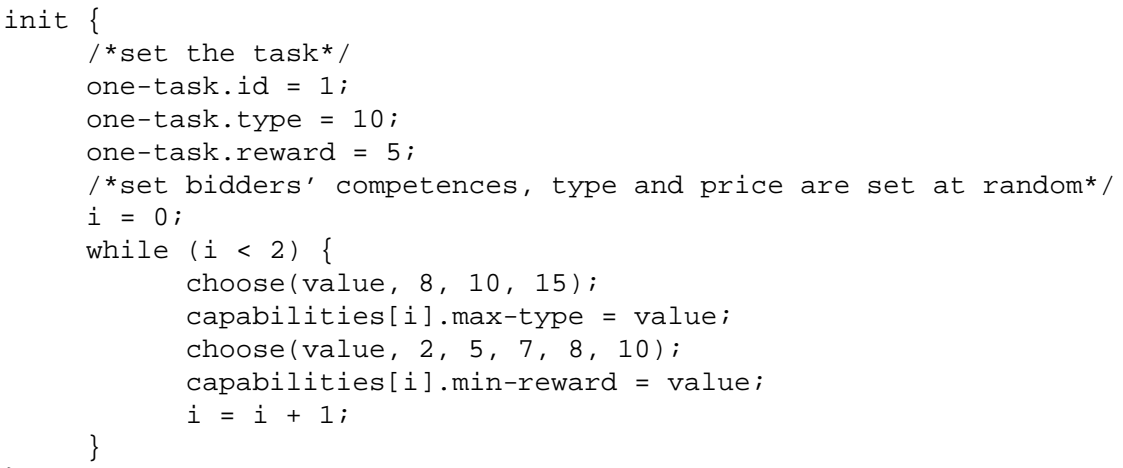

Figure 5: Initialisation for the Contract Net.

rejection. As soon as the task manager has received all answers, it selects at most one bidder to process the task. The TaskManager uses the select function to choose which agent to award the task to. Finally, the task manager sends a message to the successful bidder.

The implementation of bidding agents is shown in Figure 7. The first action of the bidders is to wait for the task announcement. Then, they call the decision function to determine if they are able to do the task. The decision is made on the basis of the task type and the reward.

We have two functions in the Contract Net implementation: the select function, used by the task manager to select a bidder to perform the task; and the decision function, used by bidders to ascertain if they are able to perform the task. The implementation of the select function is shown in Figure 8, while the decision function is shown in Figure 9.

The decision function is used by the bidders to know if they are able to perform the task. This decision is determined by the task type and the reward. If the task type is within their capabilities, and if the reward is greater or equal to their request, then they accept the task. If the task type is beyond their capabilities, they refuse the task. If the reward is less than the one expected, they accept the task but only for their requested reward.

\section{Running the example}

After writing the MABLE code, designers can execute the system: the following output was generated by MABLE when it was invoked with the Contract Net example in simulation mode ${ }^{2}$.

Bidder 0 launched!

TaskManager launched!

\footnotetext{
${ }^{2}$ Note that this is just one possible run among several.
} 


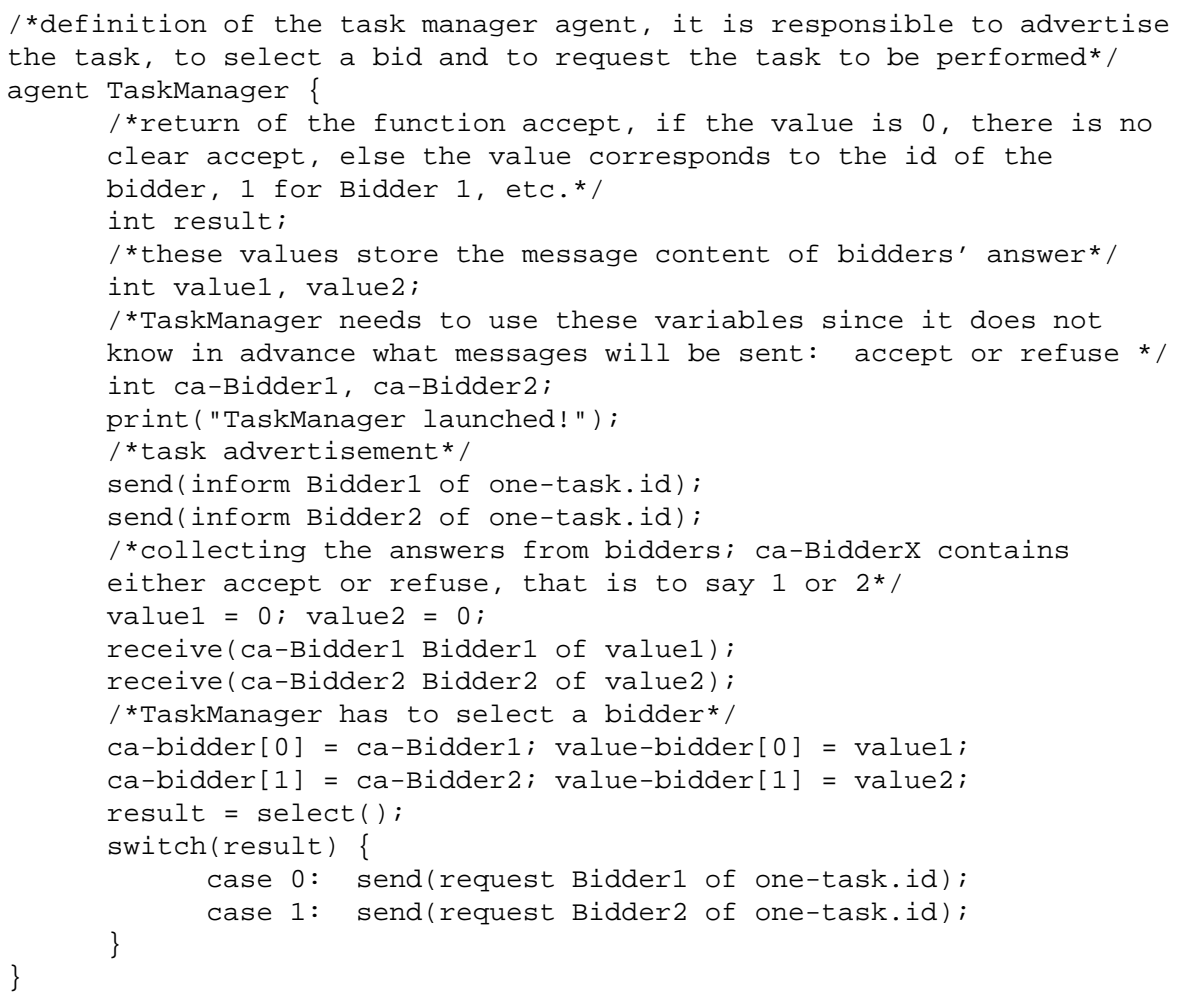

Figure 6: The Task Manager Definition.

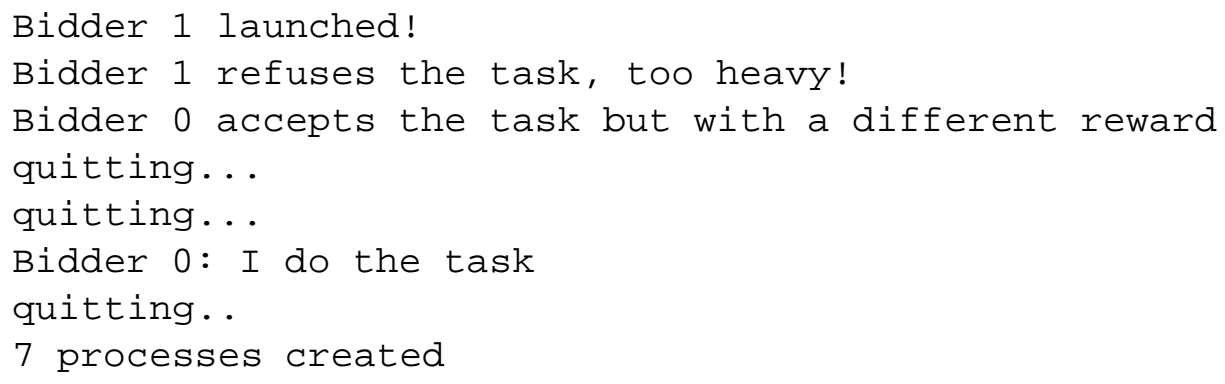

\section{Model Checking the Contract Net Protocol}

Having implemented the Contract Net in MABLE, it is natural to then use MABLE's verification capabilities to check the implementation. We will just give two properties that may be checked:

1. when the task is advertised, eventually it will be awarded to some agent;

2. when the task is advertised, eventually it will be performed at a different reward. As stated in section 2.3, properties have to be expressed as claims to be checked. The first property gives the following claim: 


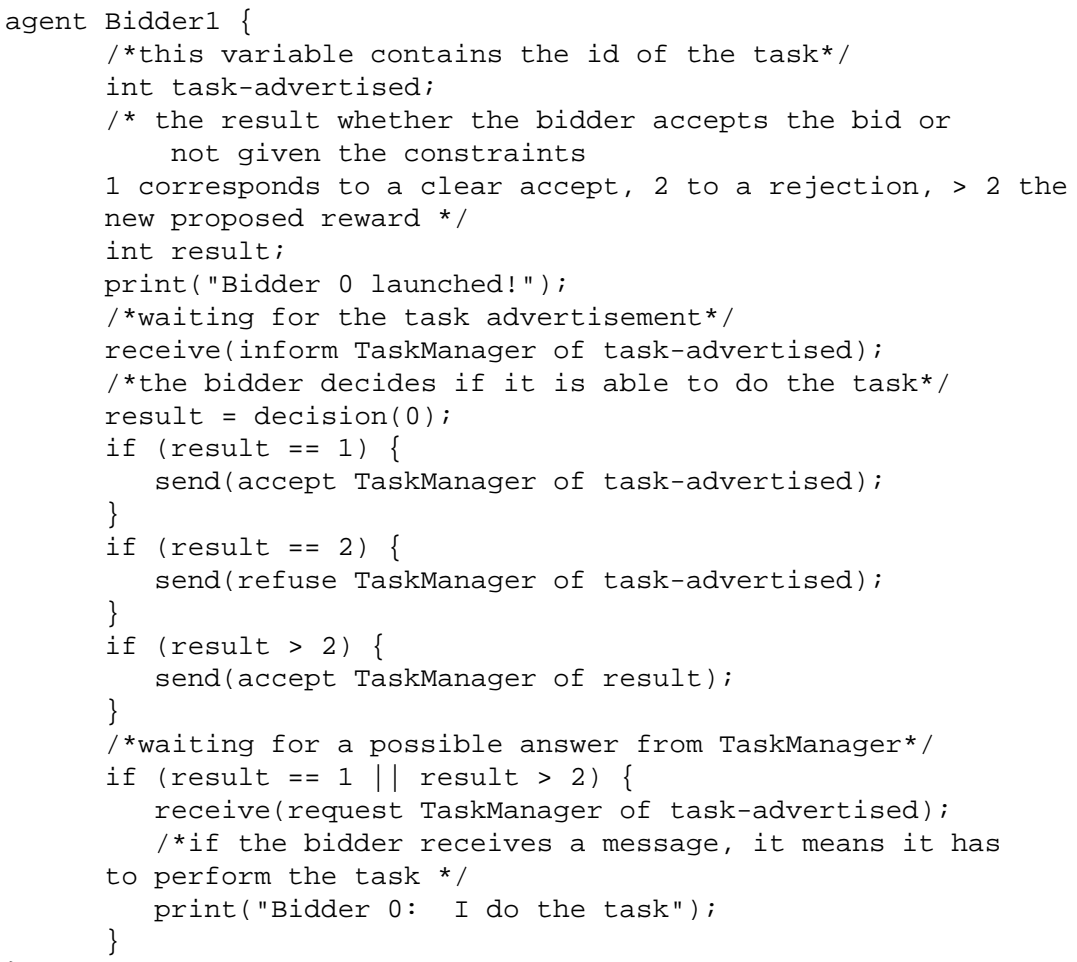

Figure 7: The Bidder Definition.

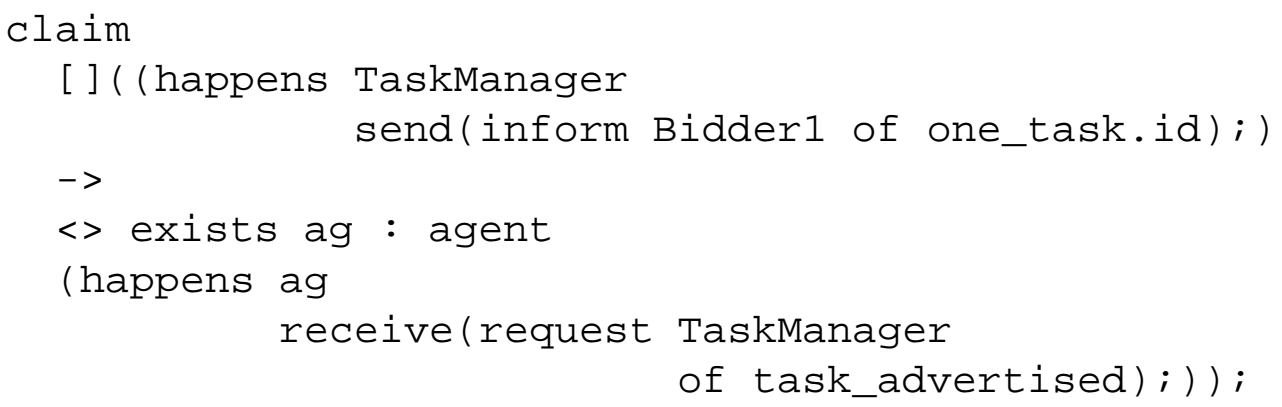

The second property corresponds to the following claim.

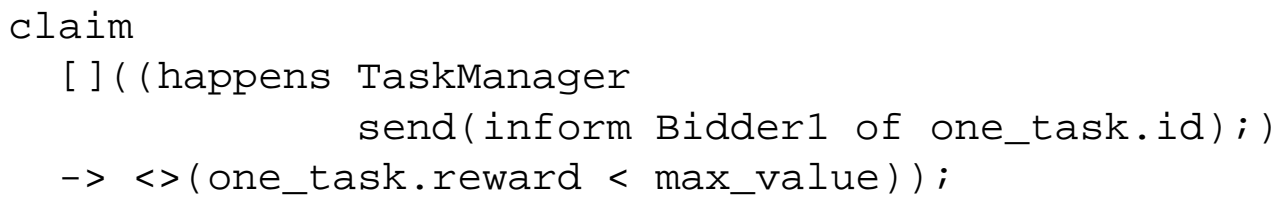

It took about six minutes on a PC with an Intel Pentium III $500 \mathrm{MHz}$ processor and $256 \mathrm{Mb}$ RAM to verify each of these results. 


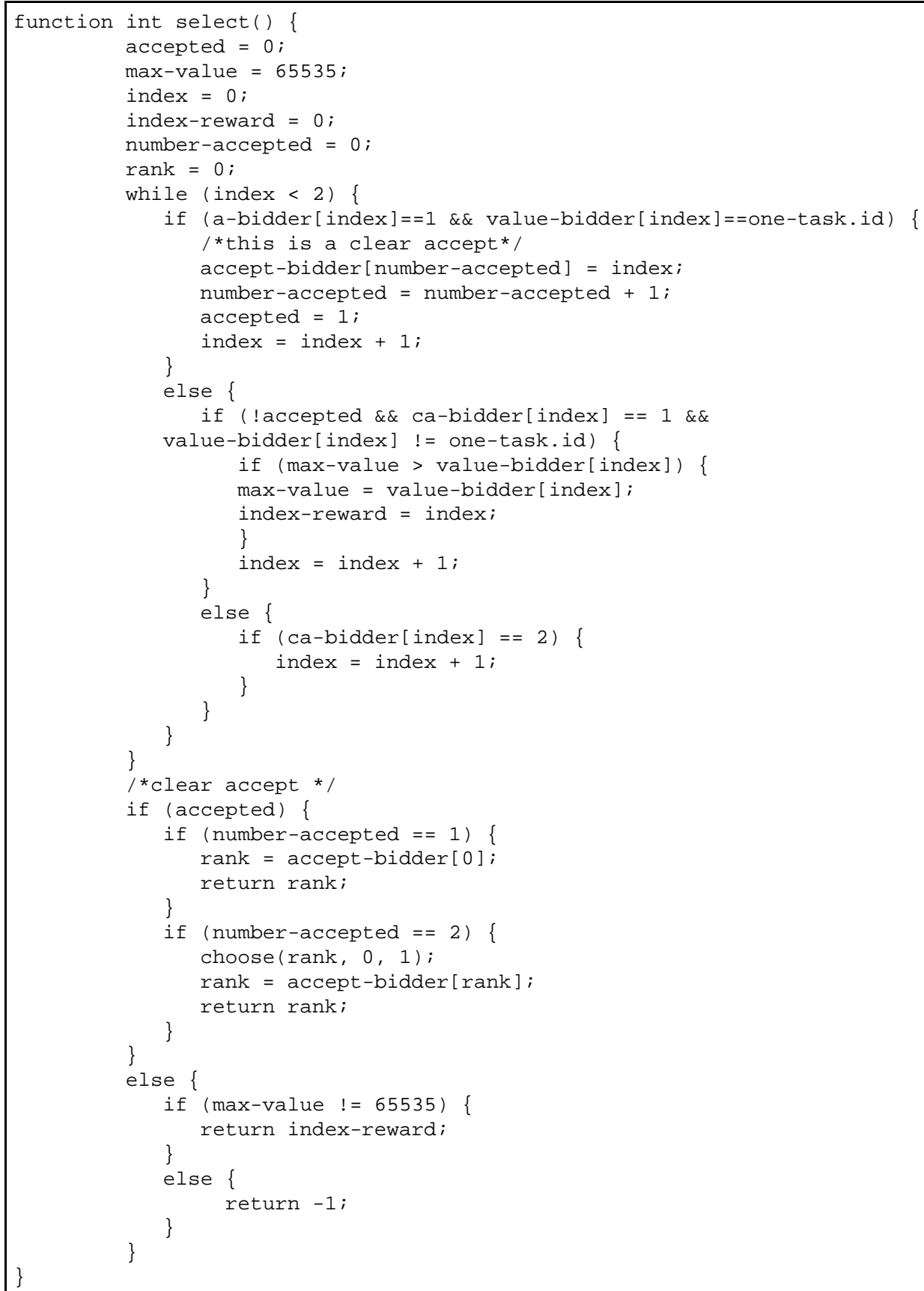

Figure 8: The Select Function. 


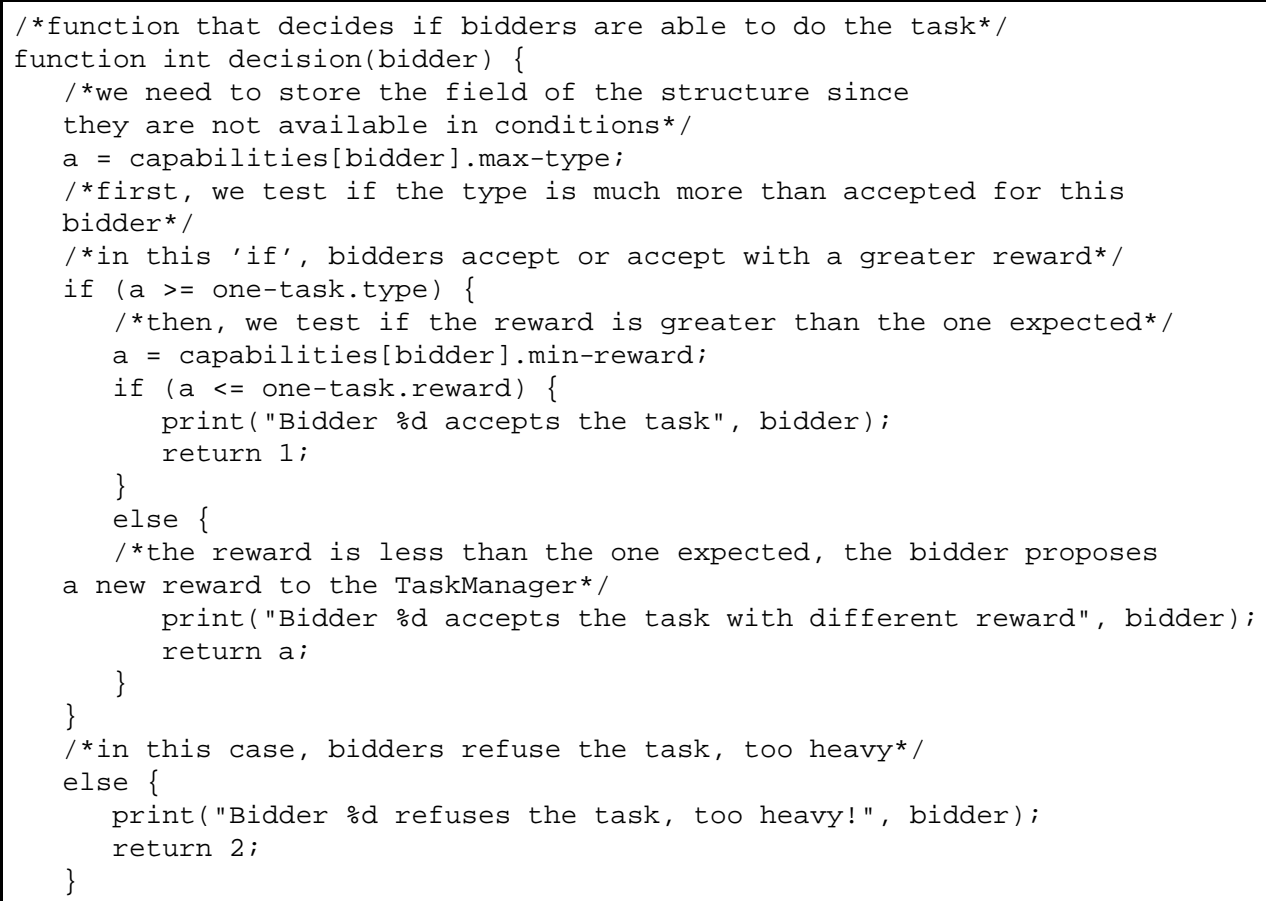

Figure 9: The Decision Function.

\section{The mable Compiler}

In this section, we give a brief overview of the way in which the MABLE compiler works. The compiler translates MABLE systems into a form that can be processed by the SPIN model checker $[16,17]$. The way in which the MABLE compiler interacts with SPIN is illustrated in Figure 10.

There are four key components to the MABLE compiler: the way in which individual agents and their control constructs (e.g., loops) are translated to PROMELA; the way in which belief-desire-intention states are implemented; the way in which $\mathcal{M O R} \mathcal{A}$ claims are dealt with; and the way in which performative semantics are dealt with.

\section{Agents and Basic Control Structures}

Dealing with the basic MABLE control constructs is straightforward. Although PROMELA is a relatively low-level language, it is straightforward to map MABLE's control constructs into those provided by PROMELA. Agents in MABLE are implemented as processes (proctypes) in PROMELA; additional PROMELA initialisation code is generated to (automatically) start agents simultaneously. 


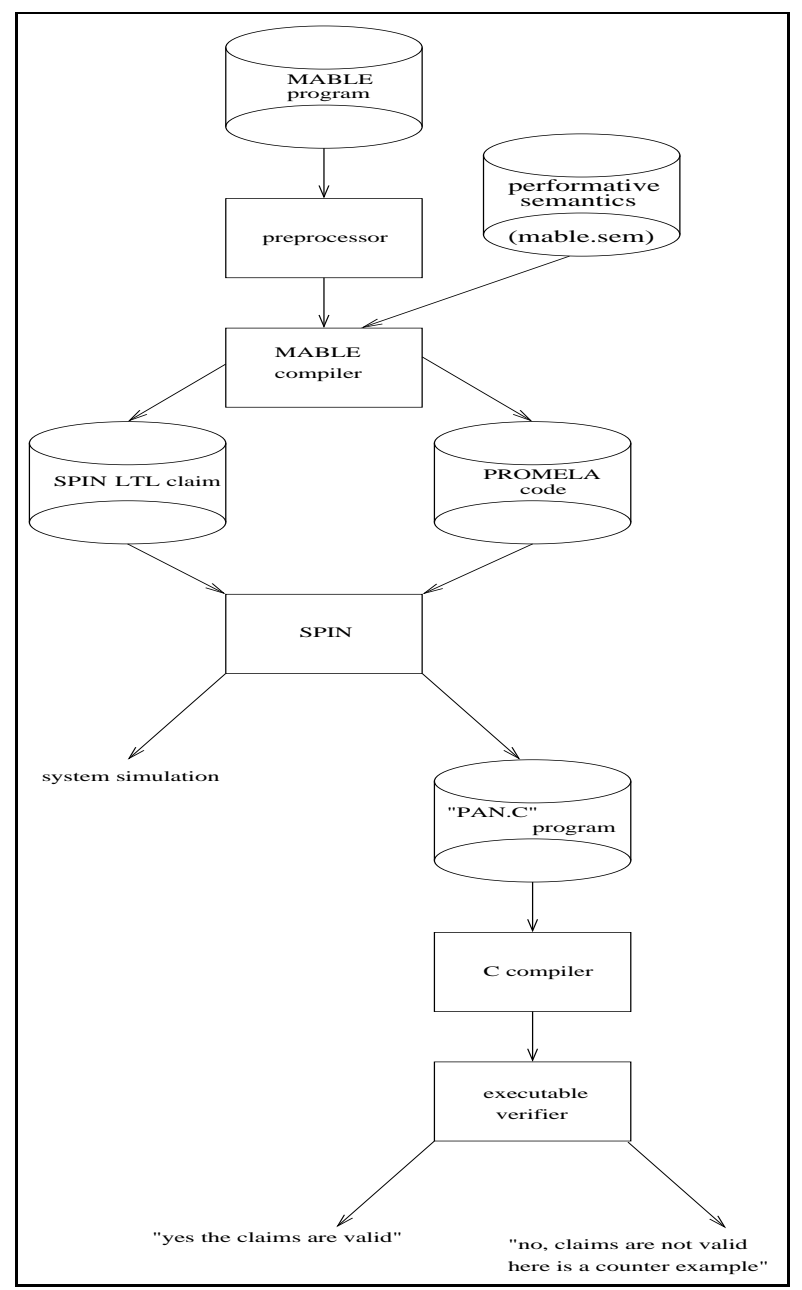

Figure 10: Operation of the MABLE system. 


\section{Mental States}

More interesting is the way that mental states are dealt with. The idea is to model these as finitely nested data structures (in the style of $[18,2]$ ). Predicates are represented by propositional abstraction: where a predicate appears in the context of a modality, a new proposition symbol is introduced to represent this predicate. This new proposition is then used in the BDI data structures. A key disadvantage of this approach is that it causes a blow-up in the size of the state space. For this reason, nested mental states are only generated on an "as needed" basis.

\section{Claims}

To implement claims, we need to map $\mathcal{M O R} \mathcal{A}$ formulae into the LTL form accepted by SPIN. In this mapping, we need to deal with a number of features that are not supported directly by LTL:

- Quantifiers are removed by expansion. Quantification is over finite domains, and so any quantified formula can be rewritten into a quantifier-free formula by expanding universal quantification into a conjunction, and existential quantification into a disjunction.

- BDI modalities are removed by replacing them with predicates about the corresponding data structures in the implemented system.

- Predicates are removed by propositional abstraction: each predicate is replaced by proposition, the truth of which is bound to the predicate it replaces.

- To deal with happens operators, we insert new code into the program itself, flagging the occurrence of statements that occur in happens operators. These flags can then be referred to in the LTL formulae generated by MABLE. Suppose we have an operator (happens ag $s$ ) occurring in a claim. First, MABLE replaces this operator in the claim with a new proposition, say $\mathrm{p}$. The MABLE compiler then passes over the parse tree of the MABLE program for agent ag, looking for the statement $s$ in the program code. Whenever it finds $s$ in the parse tree, it inserts a new statement into the program immediately before $s$, setting the corresponding new proposition $\mathrm{p}$ to true; and following the statement $\mathrm{s}$, another new program statement is inserted, setting the proposition $p$ to false. The toggling of the proposition $\mathrm{p}$ is wrapped within PROMELA atomic constructs, to ensure that the toggling process itself does not alter the control flow of the generated system. In this way, the truth of the proposition $\mathrm{p}$ indicates that the next statement to be executed by ag is $\mathrm{s}$.

The end result is a propositional LTL formula, suitable for input to the SPIN model checker, together with a list of predicates and the names of the propositions with which they were replaced. Together with the generated PROMELA code, these can be fed directly into SPIN for checking. 


\section{Performative Semantics}

Recall that it is possible to define the semantics of performatives separately from the MABLE system itself, using the mable. sem file. The MABLE compiler looks for such a file containing a number of performative definitions, where each performative definition has the following structure:

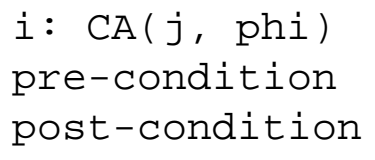

where $i, j$ and phi are the sender, recipient, and content of the message respectively, and $C A$ is the name of the performative. These semantic definitions are dealt with as follows.

With respect to the pre-condition, suppose that a particular agent contained a send statement with the performative $C A$. Then this send would be translated into a PROMELA guarded command with the following structure.

$$
\text { pre-condition }->\text { send the message }
$$

The "->" is PROMELA's guarded command structure: to the left of $->$ is a condition, and to the right is a program statement (an action). The semantics of this construct are that the process executing this statement will suspend (in effect, go to sleep) until the condition on the left hand side is true. When (more accurately, if) the condition becomes true, then the right hand side is "enabled": that is, it is ready to be executed, and assuming a fair process scheduler, will indeed be executed.

With respect to the post-condition, suppose an agent contained a receive statement with the performative CA. Then this receive statement would be translated into PROMELA code with the following structure.

receive message;

make post-condition true

Thus once a message is received, the post-condition will be asserted.

\section{Related Work}

In recent years, a number of logic-oriented multi-agent programming languages have been developed, which attempt to bring logics of rational agency somewhat closer to programming languages. In this section, we will briefly consider the relationship of MABLE to this work.

Perhaps the best-known multiagent language is AGENT0 [33]. Developed by Shoham in the late 1980s, this was the first language to be explicitly referred to as 
an "agent-oriented programming" language; additionally, Shoham was keen to link AGENT0 to a multimodal logic of rational agency [39]. The logic was clearly intended to provide a logical semantics for the language, although the precise details were never made formal. The programming model for AGENT0 was that of rule-based systems: one programmed an agent in terms of a set of commitment rules, which defined how an agent formed and discharged commitments to action. Agents communicated with one-another using three performatives: request, unrequest, and inform. The semantics of these performatives was not really hardwired into the language: a programmer defined their effects by writing rules to handle them.

A number of later languages were developed in the AGENT0 mould. The PLACA language, adopting a rule-based programming model very similar to that of AGENT0, was intended to overcome a number of deficiencies of AGENT0, such as the ability of agents to explicitly plan how to meet their commitments [38].

Rao's AGENTSPEAK was another influential agent-oriented programming language [28]. Agents in AGENTSPEAK are programmed by defining a set of plans (somewhat like Shoham's commitment rules), which are executed both reactively (plans are invoked in response to events in the environment), and pro-actively (plans can be explicitly invoked by other plans). This programming model is essentially a distillation of Georgeff and Lansky's Procedural Reasoning System (PRS) [13], a reactive planning framework developed in the mid-1980s that subsequently formed the basis of several other agent-oriented programming systems, notably DMARS [8]. Rao and Georgeff developed a number of BDI logics, which, like Shoham's logics, were ultimately intended to provide a semantics to AGENTSPEAK and the PRS [29]. However, as with Shoham's language, while there was plenty of intuition about how the logics related to the programming language [30], the precise relationship between language and logic was never made formal. As a consequence, one could never really claim that a formula of the logic expressed a property of a system; and as a consequence, one could never verify whether the formula represented a property that was true or false of a given system. It is worth noting that Rao and Georgeff developed preliminary model checking techniques for BDI logics [31], although because the relationship between the logic and the PRS/AGENTSPEAK was never made precise, these model checking techniques could not be deployed to verify PRS/AGENTSPEAK systems [40, 43]. (It is worth noting that at the time of writing, work is underway to develop techniques for model checking AGENTSPEAK systems [3].) Note that Hindriks' 3 APL language was a direct descendant of AGENSPEAK [15]. In 3 APL, agents are also programmed using a rule-like model; the semantics and proof theory of 3 APL were developed in some detail.

GOLOG [21, 32] and its multiagent sibling CONGOLOG [20] represent another rich seam of work on logic-oriented approaches to programming rational agents. Essentially, GOLOG is a framework for executing a fragment of the situation calculus; the situation calculus is a well known logical framework for reasoning about action [23]. Put crudely, writing a GOLOG program involves expressing a logical theory of what action an agent should perform, using the situation calculus; this theory, together with 
some background axioms, represents a logical expression of what it means for the agent to do the right action. Executing such a program reduces to constructively solving a deductive proof problem, broadly along the lines of showing that there is a sequence of actions representing an acceptable computation according to the theory [32, p.121]; the witness to this proof will be a sequence of actions, which can then be executed.

A closely related approach is that of the METATEM paradigm [12]. In METATEM, an agent is programmed by giving it a temporal logic specification of the behaviour it should exhibit, where this specification is a conjunction of past $\Rightarrow$ future rules. The process of executing the specification corresponds to doing a constructive proof of the satisfiability of the program formula, where the model being constructed is built in part by the agent, and part by the environment. Another somewhat related language is the IMPACT framework of Subrahmanian et al. [37]. IMPACT is a rich framework for programming agents, which draws upon and considerably extends some ideas from logic programming. Agents in IMPACT are programmed by using rules that incorporate deontic modalities (permitted, forbidden, obliged [25]). These rules can be interpreted to determine the actions that an agent should perform at any given moment [37, p.171].

A common feature of all the languages mentioned in this section is that the reasoning used to determine the action to perform takes place at run time. Moreover, as we are in all cases essentially executing a logical formula, the issue of verification does not really arise - for example, we can be assured that any execution trace of a METATEM program is a model of the program formula. Indeed, this is one of the key arguments in favour of using an executable logic framework: we can be assured that a logical program will execute according to its semantics.

There are, however, several disadvantages to deciding what action to perform by reasoning at run time. The most obvious of these is that reasoning is generally computationally costly: see, for example, the complexity results associated with algorithms for the IMPACT framework [37, pp.399-460]. A more subtle problem is that requiring a programmer to express a program in the language of logic (be it situation calculus, temporal logic, or deontic logic) is often not desirable. Ideally, we want to be able to let the programmer use their most preferred programming tools, and then verify their work, rather than imposing a programming regime on them. In this sense, we believe, MABLE is closer to the reality of everyday programming. It provides constructs corresponding to those that programmers everywhere are familiar with, enriched with agent-oriented constructs. As verification is done at design time, the issue of run-time reasoning (and all the potential difficulties it entails) does not arise. The disadvantage of the MABLE approach is of course that we lose the elegant logical semantics associated with directly executing logical formulae; but we argue that as we can directly verify MABLE systems, this is not a major issue. 


\section{Conclusions}

In this paper, we have described the MABLE language for multiagent systems. This fully-implemented language supports the development of agents in an imperative programming language, enriched by some features from the agent-oriented programming paradigm. However, the most important (and novel) feature of MABLE is that it supports the automatic verification of MABLE systems via model checking. A designer can formally express the requirements of MABLE systems as formulae of linear-time BDI logic, and MABLE is capable of automatically verifying whether or not the system does or does not satisfy these requirements. Another novel feature is that although the key communication mechanism in MABLE is asynchronous message passing in the style of FIPA and KQML, MABLE does not dictate a semantics for the performatives used in communication. Instead, a designer can explicitly define the semantics of performatives separately from a system and, in this way, can explore the behaviour of the same system for a range of performative semantics. Combining this feature with the model checking capabilities of MABLE, it becomes possible to automatically verify compliance to agent communication language performatives - a problem of some interest to the agent communication language community.

There are a number of obvious avenues for future research. First, we hope to further extend the language and its model checking facilities. For example, it would be useful to add features such as true unification to the language, and other similar reasoning features. However, the addition of such features will inevitably lead to a (further) blow-up in the state space of the generated system. For this reason, we intend to study the possibility of automatic abstraction of MABLE systems: essentially, stripping MABLE systems down to their leanest possible representation. Another issue we are pursuing is that of automatically generating JAVA code from MABLE systems. For example, suppose we have a MABLE system that we have verified complies with the semantics of the FIPA language. Then automatically generating JAVA code that implements the FIPA performatives via the JADE implementation of the FIPA language [27], we can plausibly (if not entirely accurately) claim that the resultant JAVA system respects the FIPA semantics.

\section{References}

[1] R. Alur, L. de Alfaro, T. A. Henzinger, S. C. Krishnan, F. Y. C. Mang, S. Qadeer, S. K. Rajamani, and S. Taşiran. MOCHA user manual. University of Berkeley Report, 2000.

[2] M. Benerecetti, F. Giunchiglia, and L. Serafini. A model checking algorithm for multiagent systems. In J. P. Müller, M. P. Singh, and A. S. Rao, editors, Intelligent Agents V(LNAI Volume 1555). Springer-Verlag: Berlin, Germany, 1999. 
[3] R. H. Bordini, M. Fisher, C. Pardavila, and M. Wooldridge. Model checking agentspeak. In Proceedings of the Second International Conference on $\mathrm{Au}$ tonomous Agents and Multiagent Systems (AAMAS-03), Columbia University, NY, USA, July 2003.

[4] E. M. Clarke, O. Grumberg, and D. A. Peled. Model Checking. The MIT Press: Cambridge, MA, 2000.

[5] W. F. Clocksin and C. S. Mellish. Programming in Prolog. Springer-Verlag: Berlin, Germany, 1981.

[6] P. R. Cohen and C. R. Perrault. Elements of a plan based theory of speech acts. Cognitive Science, 3:177-212, 1979.

[7] F. Dignum and M. Greaves, editors. Issues in Agent Communication (LNAI Volume 1916). Springer-Verlag: Berlin, Germany, 2000.

[8] M. d'Inverno, D. Kinny, M. Luck, and M. Wooldridge. A formal specification of dMARS. In M. P. Singh, A. Rao, and M. J. Wooldridge, editors, Intelligent Agents IV (LNAI Volume 1365), pages 155-176. Springer-Verlag: Berlin, Germany, 1997.

[9] FIPA. Specification part 2 - Agent communication language, 1997. The text refers to the specification dated 23 October 1997.

[10] FIPA. Specification part 2 - Agent communication language, 1999. The text refers to the specification dated 16 April 1999.

[11] FIPA. The foundation for intelligent physical agents, 2001. See http://www.fipa.org/.

[12] M. Fisher. A survey of Concurrent MetateM - the language and its applications. In D. M. Gabbay and H. J. Ohlbach, editors, Temporal Logic - Proceedings of the First International Conference (LNAI Volume 827), pages 480-505. Springer-Verlag: Berlin, Germany, July 1994.

[13] M. P. Georgeff and A. L. Lansky. Reactive reasoning and planning. In Proceedings of the Sixth National Conference on Artificial Intelligence (AAAI-87), pages 677-682, Seattle, WA, 1987.

[14] J. Y. Halpern and Y. Moses. A guide to completeness and complexity for modal logics of knowledge and belief. Artificial Intelligence, 54:319-379, 1992.

[15] K. V. Hindriks, F. S. de Boer, W. van der Hoek, and J.-J. Ch. Meyer. Agent programming in 3APL. Autonomous Agents and Multi-Agent Systems, 2(4):357402, 1999. 
[16] G. Holzmann. Design and Validation of Computer Protocols. Prentice Hall International: Hemel Hempstead, England, 1991.

[17] G. Holzmann. The Spin model checker. IEEE Transactions on Software Engineering, 23(5):279-295, May 1997.

[18] K. Konolige. A Deduction Model of Belief. Pitman Publishing: London and Morgan Kaufmann: San Mateo, CA, 1986.

[19] Y. Labrou and T. Finin. Semantics and conversations for an agent communication language. In Proceedings of the Fifteenth International Joint Conference on Artificial Intelligence (IJCAI-97), pages 584-591, Nagoya, Japan, 1997.

[20] Y. Lésperance, H. J. Levesque, F. Lin, D. Marcu, R. Reiter, and R. B. Scherl. Foundations of a logical approach to agent programming. In M. Wooldridge, J. P. Müller, and M. Tambe, editors, Intelligent Agents II (LNAI Volume 1037), pages 331-346. Springer-Verlag: Berlin, Germany, 1996.

[21] H. Levesque, R. Reiter, Y. Lespérance, F. Lin, and R. Scherl. Golog: A logic programming language for dynamic domains. Journal of Logic Programming, 31:59-84, 1996.

[22] J. Mayfield, Y. Labrou, and T. Finin. Evaluating KQML as an agent communication language. In M. Wooldridge, J. P. Müller, and M. Tambe, editors, Intelligent Agents II (LNAI Volume 1037), pages 347-360. Springer-Verlag: Berlin, Germany, 1996.

[23] J. McCarthy and P. J. Hayes. Some philosophical problems from the standpoint of artificial intelligence. In B. Meltzer and D. Michie, editors, Machine Intelligence 4, pages 463-502. Edinburgh University Press, 1969.

[24] K. L. McMillan. Symbolic Model Checking. Kluwer Academic Publishers: Dordrecht, The Netherlands, 1993.

[25] J.-J. Ch. Meyer and R. J. Wieringa, editors. Deontic Logic in Computer Science - Normative System Specification. John Wiley \& Sons, 1993.

[26] J. Pitt and E. H. Mamdani. A protocol-based semantics for an agent communication language. In Proceedings of the Sixteenth International Joint Conference on Artificial Intelligence (IJCAI-99), Stockholm, Sweden, August 1999.

[27] Agostino Poggi and Giovanni Rimassa. Adding extensible synchronization capabilities to the agent model of a FIPA-compliant agent platform. In P. Ciancarini and M. Wooldridge, editors, Agent-Oriented Software Engineering - Proceedings of the First International Workshop AOSE-2000 (LNCS Volume 1957), pages 307-322. Springer-Verlag: Berlin, Germany, 2001. 
[28] A. S. Rao. AgentSpeak(L): BDI agents speak out in a logical computable language. In W. Van de Velde and J. W. Perram, editors, Agents Breaking Away: Proceedings of the Seventh European Workshop on Modelling Autonomous Agents in a Multi-Agent World, (LNAI Volume 1038), pages 42-55. Springer-Verlag: Berlin, Germany, 1996.

[29] A. S. Rao and M. Georgeff. Decision procedures for BDI logics. Journal of Logic and Computation, 8(3):293-344, 1998.

[30] A. S. Rao and M. P. Georgeff. An abstract architecture for rational agents. In C. Rich, W. Swartout, and B. Nebel, editors, Proceedings of Knowledge Representation and Reasoning (KR\&R-92), pages 439-449, 1992.

[31] A. S. Rao and M. P. Georgeff. A model-theoretic approach to the verification of situated reasoning systems. In Proceedings of the Thirteenth International Joint Conference on Artificial Intelligence (IJCAI-93), pages 318-324, Chambéry, France, 1993.

[32] R. Reiter. Knowledge in Action. The MIT Press: Cambridge, MA, 2001.

[33] Y. Shoham. Agent-oriented programming. Artificial Intelligence, 60(1):51-92, 1993.

[34] M. Singh. Agent communication languages: Rethinking the principles. IEEE Computer, pages 40-49, December 1998.

[35] R. G. Smith. The contract net protocol. IEEE Transactions on Computers, C29(12), 1980.

[36] R. G. Smith. A Framework for Distributed Problem Solving. UMI Research Press, 1980.

[37] V. S. Subrahmanian, P. Bonatti, J. Dix, T. Eiter, S. Kraus, F. Ozcan, and R. Ross. Heterogeneous Agent Systems. The MIT Press: Cambridge, MA, 2000.

[38] S. R. Thomas. The PLACA agent programming language. In M. Wooldridge and N. R. Jennings, editors, Intelligent Agents: Theories, Architectures, and Languages (LNAI Volume 890), pages 355-369. Springer-Verlag: Berlin, Germany, January 1995.

[39] S. R. Thomas, Y. Shoham, A. Schwartz, and S. Kraus. Preliminary thoughts on an agent description language. International Journal of Intelligent Systems, 6:497-508, 1991.

[40] M. Wooldridge. Agent-based software engineering. IEE Proceedings on Software Engineering, 144(1):26-37, February 1997. 
[41] M. Wooldridge. Verifiable semantics for agent communication languages. In Proceedings of the Third International Conference on Multi-Agent Systems (ICMAS98), pages 349-365, Paris, France, 1998.

[42] M. Wooldridge. Verifying that agents implement a communication language. In Proceedings of the Sixteenth National Conference on Artificial Intelligence (AAAI-99), pages 52-57, Orlando, FL, July 1999.

[43] M. Wooldridge. Computationally grounded theories of agency. In Proceedings of the Fourth International Conference on Multi-Agent Systems (ICMAS-2000), pages 13-20, Boston, MA, 2000.

[44] M. Wooldridge. Reasoning about Rational Agents. The MIT Press: Cambridge, MA, 2000.

[45] M. Wooldridge. An Introduction to Multiagent Systems. John Wiley \& Sons, 2002. 